\title{
Age differences in working memory- The roles of storage and selective access
}

\author{
KLAUS OBERAUER, MIRKO WENDLAND, and REINHOLD KLIEGL \\ University of Potsdam, Potsdam, Germany
}

\begin{abstract}
Twenty-four young (23 years) and 24 old (71 years) adults performed arithmetic tasks with working memory loads ranging from 1 to 4 . Age groups were equivalent in mean accuracy and speed of arithmetic operations under minimal working memory load, but old adults were slower than young with memory demands $>1$. Access to a new object in working memory as the basis of computation required additional time. This object-switching cost increased with increases in memory demand, but was unaffected by age, indicating that old adults have no deficit in selective access to working memory.
\end{abstract}

Working memory is commonly conceived as a system that briefly maintains a small number of representational elements, providing access to them for processing (see, e.g., Baddeley, 1986; Cowan, 1999; Oberauer, 2002). In addition, working memory is associated with executive functions that control and supervise ongoing processes (Baddeley, 1996; Engle, Tuholski, Laughlin, \& Conway, 1999). In comparison with young adults, old adults are known to have reduced working memory capacity (e.g., Craik, Anderson, Kerr, \& Li, 1995; Salthouse, 1994). The main goal of the present study is to dissociate two functions of working memory-maintenance of information and selective access to it for processing — and to investigate which of these functions is responsible for old adults' impairments in tasks with high working memory demands.

General factor theories of cognitive aging view this impairment as a by-product of a more basic deficit, general mental slowing in older age (Fisk \& Warr, 1996; Salthouse, 1996). Several studies, however, have shown that old adults' problems with tasks taxing working memory go beyond what can be expected from their performance in comparable tasks that place less demand on working memory (Kliegl, Mayr, \& Krampe, 1994; Mayr \& Kliegl, 1993; Mayr, Kliegl, \& Krampe, 1996). Mayr and Kliegl (1993) introduced the concepts of sequential and coordinative complexity to capture the difference between tasks where old adults are just generally slowed and those where they have additional problems beyond general slowing.

\section{Sequential and Coordinative Complexity}

Tasks with sequential complexity can be solved by a sequence of cognitive operations, such that each operation requires access only to the result of its immediate prede-

This research was supported by Deutsche Forschungsgemeinschaft (DFG, Grant KL 955/4). We thank Steven Hecht, Patrick Lemaire, and Jane Zbrodoff for valuable comments on an earlier version of the article. We also thank Petra Grüttner for collecting the data of the older participants. Correspondence should be addressed to K. Oberauer, Department of Psychology, University of Potsdam, P. O. Box 6015 53, 14415 Potsdam, Germany (e-mail: ko@ ry.uni-potsdam.de). cessor. Thus, there is no need to remember intermediate results in working memory and to retrieve them at a later point. A typical example of a task with sequential complexity is an arithmetic chain involving only single-digit numbers and no brackets. Tasks with coordinative complexity, in contrast, require storage of and access to intermediate results in working memory. Examples from mental arithmetic are tasks involving brackets or carries.

In a study by Verhaeghen, Kliegl, and Mayr (1997), old and young adults solved simple arithmetic chain tasks with equal speed and accuracy. On chain tasks including brackets, however, old adults were slower and less accurate than the young adults. This finding points to a specific age-related deficit in the complex tasks that cannot be reduced to age differences in speed or accuracy of basic arithmetic operations. The study by Verhaeghen et al. is particularly revealing, because it demonstrates age-related deficits in complex tasks in the presence of age invariance in a simpler version of the same task.

Since tasks with coordinative complexity differ from those with sequential complexity in their demand on working memory, it seems reasonable to assume that old adults' reduced working memory capacity is responsible for their specific problems with coordinative tasks. The goal of our study is to attribute these problems more precisely to a specific function of working memory. The studies reviewed above leave open at least three possibilities:(1) Tasks with coordinative complexity could be difficult for old adults because they place a higher load on the memory function of working memory; that is, they require more information elements to be remembered simultaneously during task solution. Mental arithmetic tasks with brackets, for example, require that people solve the equation within a bracket while at the same time remembering the intermediate result reached so far. (2) Tasks with coordinative complexity typically require selective access to the contents of working memory. Intermediate results in arithmetic tasks, for example, must be picked up for further computation at later steps (e.g., when the brackets are solved). The problems of older adults with these tasks 
could arise from the need to access the right information in working memory at the right time. This could be particularly difficult when the task requires frequent switching between different working memory contents that must be selected for processing. (3) It could be the executive demands associated with coordinative tasks that pose specific problems for old adults (Mayr et al., 1996). Whereas every single processing step in a task with sequential complexity is usually guided by an imperative stimulus (e.g., the next addend or subtrahend in a chain), in tasks with coordinative complexity people must typically sequence several cognitive operations on their own in an appropriate manner (e.g., decide to solve the innermost bracket first).

\section{Memory Load and Memory Access}

In the present study, we varied independently the first two factors mentioned above, memory load and memory access, while attempting to hold the third factor constant by minimizing executive demands such as self-guided scheduling of operations. In a previous study (Oberauer, Demmrich, Mayr, \& Kliegl, 2001), we used a dual-task paradigm to investigate age-differential effects of a concurrent memory load on mental arithmetic under two conditions: In the memory load condition, participants solved arithmetic chain tasks while holding an unrelated string of digits in working memory. In the access condition, participants had to retrieve digits from the memory set to replace variables in the arithmetic task. Although an unrelated load did not affect the efficiency of arithmetic processing relative to a single-task control, access to the memory set drastically impaired speed and accuracy of mental arithmetic. When load and access condition were equated in their executive demands (Oberauer et al., 2001, Experiment 2), the access demand affected young and old participants to the same degree. This suggests that there is no specific age-related deficit in selective access to working memory. On the other hand, old adults forgot more digits while doing the arithmetic task, pointing to a potential deficit in the memory function of working memory. With the study reported here, we intended to validate and extend this conjecture with a different experimental paradigm.

We used an arithmetic memory-updating task similar to that in Oberauer and Kliegl (2001). Participants memorized from one to four initial digits, with each digit assigned to a frame on the screen. Then they updated individual digits according to arithmetic operations displayed in the respective frames (see Figure 1). The task version with a single frame is a task with sequential complexity (i.e., an arithmetic chain task). Coordinative complexity comes into play when there is more than one frame: While one digit is processed, one or more other digits must be remembered, introducing a load on the memory function of working memory that increases as the number of frames increases.

Moreover, successive updating steps sometimes require access to digits in different frames, thus introducing the demand to switch between objects in working memory. Switching from one object to another in working memory is associated with a substantial time cost (Garavan, 1998). By independently varying memory load (i.e., the number

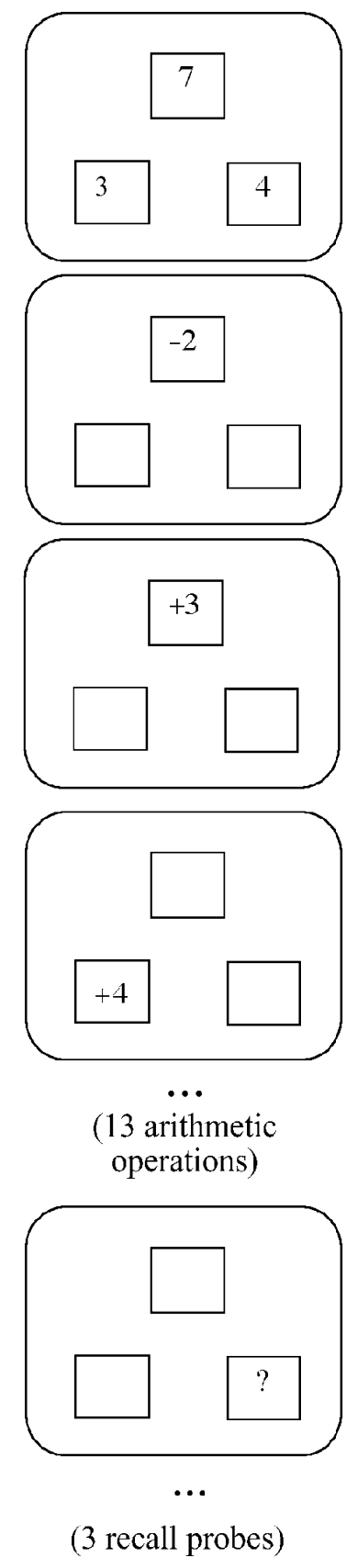

Figure 1. Example task of numerical memory updating with memory demand 3. Successive computer displays are shown from top to bottom. The display of initial digits is followed by 13 arithmetic operations, after which final results are probed by three successive question marks in the three frames. The second operation shown is a nonswitch operation; the third one is a switch operation. Each successive operation is triggered by pressing the space bar.

of frames) and switching between objects in working memory (i.e., accessing the same frame as before vs. a different frame for updating), we can disentangle the contributions of load and access to the specific difficulty associated with coordinative complexity. 


\section{Age Invariance in Mental Arithmetic?}

We chose mental arithmetic as our basic task because retrieval of arithmetic facts-or of lexical knowledge in general - has been suggested as a domain of age invariance (Kliegl, Mayr, \& Oberauer, 2000). One hint in this direction comes from studies showing no age difference in the problem-size effect (Allen, Ashcraft, \& Weber, 1992; Allen, Smith, Jerge, \& Vires-Collins, 1997; Geary \& Wiley, 1991; Sliwinski, Buschke, Kuslansky, Senior, \& Scarisbrick, 1994). The problem-size effect consists in an increase in latencies with the absolute size of the operands; it is interpreted as reflecting the fact retrieval component of response latencies. More compelling evidence for age invariance comes from the occasional finding of equivalent performance of young and old adults in simple arithmetic tasks (Kliegl et al., 2000; Verhaeghen et al., 1997), but this has not been observed consistently even in very similar conditions (e.g., Oberauer \& Kliegl, 2001). To foreshadow, the results of the present study constitute another case of age invariance in simple arithmetic computations.

\section{METHOD}

\section{Participants}

Twenty-four young adults (mean age $=23.3$ years, $S D=3.43$ ) and 24 old adults (mean age $=71.0$ years, $S D=3.75$ ) participated. Young adults received partial course credit or $50 \mathrm{DM}$ (about \$30); old adults were reimbursed with $90 \mathrm{DM}$. There were 12 men and 12 women in each age group. Further descriptive data for the two samples are summarized in Table 1.

\section{Materials}

Each task began with the presentation of one to four rectangular frames on the computer screen, corresponding to the memory demand. In the memory demand 1 condition, a single frame was displayed centrally; in the other conditions, the frames were arranged equidistantly on a virtual circle. When the participant pressed the space bar, the initial memory values, one in each frame, were presented simultaneously. The participants were asked to remember these values and to press the space bar when ready, after which the first operation was displayed in one of the frames. The participants had to apply the operation to the frame's current memorized value and then replace this value with the result. They pressed the space bar again when finished, thereby triggering the next operation in the same frame (a nonswitch operation) or in another frame (a switch operation, in conditions with memory demand $>1$ only). A sequence of 13 operations was performed in this way, after which all final values were probed by question marks in the respective frames in a clockwise order. The results were entered via the computer keyboard, and a high or low tone following each keypress indicated whether or not they were correct. The participants were instructed to encode the initial digits accurately and then compute the successive arithmetic operations as quickly as possible while maintaining a high level of accuracy. No time pressure was imposed on the typing of the final results.

Arithmetic operations ranged from +1 to +7 and from -1 to -7 . All intermediate and final results remained in the range from 1 to 9 . The operations +8 and -8 were excluded, because they would determine their result without the participant's knowledge of the value to which they were applied (e.g., " +8 " must always result in 9, given that the result can not exceed 9). An example task is illustrated in Figure 1 above.

For memory demands 2 to 4 , object switching was realized in two modes: In the predictable switching mode, every second operation involved a switch to a new object (i.e., a new frame), and the switch always moved clockwise around the virtual circle on which the
Table 1

Descriptive Sample Data: Means, With Standard Deviations

\begin{tabular}{lccccc}
\hline & \multicolumn{2}{c}{ Young } & & \multicolumn{2}{c}{ Old } \\
\cline { 2 - 3 } \cline { 5 - 6 } & $M$ & $S D$ & & $M$ & $S D$ \\
\hline Digit symbol substitution & 61.37 & 12.02 & & 49.5 & 7.87 \\
Vocabulary test (HAWIE) & 32.33 & 4.49 & & 34.9 & 3.4 \\
Years of formal schooling & 13.0 & 0 & & 10.6 & 2.87 \\
Physical health & 2.04 & 0.75 & & 2.26 & 0.91 \\
Mental health & 2.17 & 0.64 & & 2.09 & 0.79 \\
Cognitive fitness & 2.42 & 0.50 & & 2.08 & 0.73 \\
\hline
\end{tabular}

Note-Digit symbol substitution is a standard test for perceptual speed (a component of fluid intelligence), taken from the German version of the Wechsler intelligence test (HAWIE). The vocabulary test is an indicator of crystalline intelligence, also taken from the HAWIE. Health and fitness were self-rated on a scale from 1 (= very good $)$ to 5 (= very bad).

frames were arranged. In the random mode, the object of each operation was determined at random. Each operation was a switch or a nonswitch operation with a probability of .5 , and in case of a switch, the new object was selected at random (in conditions with memory demand $>2$ ). The predictability factor was introduced in order to separate effects of expectations from genuine costs of accessing a new object in working memory.

All tasks were generated by a computer program according to the constraints specified above. Tasks were grouped into blocks of 10 with the same memory demand and predictability mode. Within each block, the administration order of tasks was determined at random for each participant.

\section{Procedure}

The young participants were tested individually in three sessions of about $1.5 \mathrm{~h}$ each. Because we anticipated that the old adults would need more time, we split each session into two for them, resulting in six sessions of about $1 \mathrm{~h}$. During the first session (young)/the first two sessions (old), the participants practiced the memory-updating task with 10 tasks in each of the seven conditions, beginning with memory demand 1 , followed by the three predictable switch blocks in ascending order of memory demand, and then the three random switch blocks, again in ascending order of memory demand. Several additional short tests were taken during this part of the experiment, on which we will not report here. In the remaining two (four) sessions, the test blocks of memory updating were administered. One block (10 tasks) of memory demand 1 was administered at the beginning of the first test session and one at the end of the last session. In between, the participants worked through sequences of three blocks (10 tasks per block) with memory demands 2,3 , and 4 , respectively. The order of memory demands in a block triplet was counterbalanced across participants. The same sequence of three blocks was repeated four times, twice in each predictability mode. Half the participants in each age group began with the predictable switch condition, the other half with the random condition.

\section{RESULTS}

For all statistical tests, the alpha level was set to .05. A first analysis revealed that predictability of an object switch had no significant main effect on accuracy or latency, nor did it enter into any interaction. This confirms that the object switch costs are not generated or increased by participants' expectations about where the next operation will appear. As a consequence, we collapsed data over this factor for all analyses.

Percentage of correct responses at final recall was analyzed in a 2 (age) $\times 4$ (memory demand) analysis of vari- 


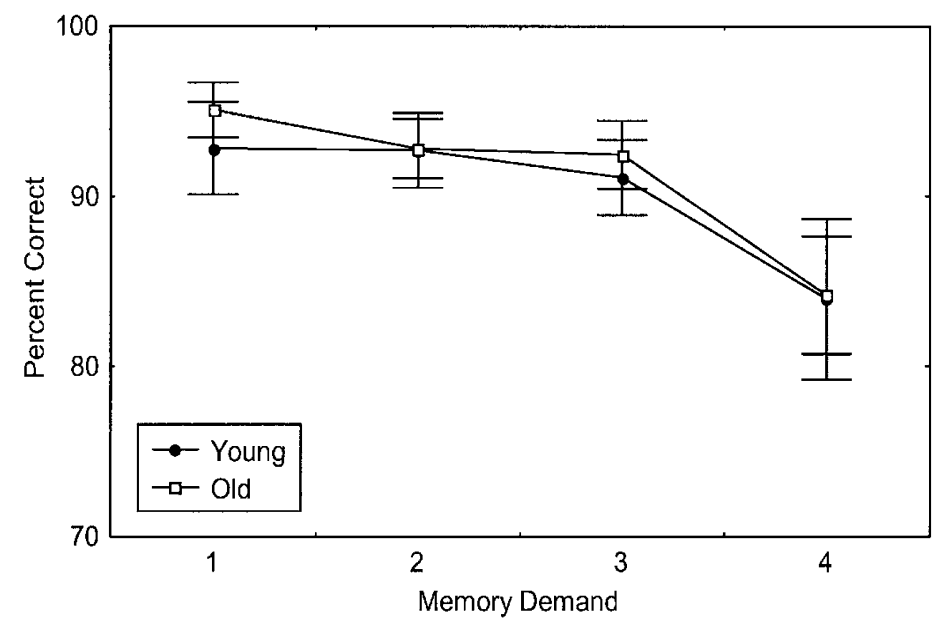

Figure 2. Mean accuracy for young and old adults. Error bars represent two standard errors.

ance (ANOVA). There was a main effect of memory demand $\left[F(3,138)=33.28, M S_{\mathrm{e}}=28.8\right]$, but no main effect of age and no interaction (both $F_{\mathrm{s}}<1$ ). Figure 2 shows an accelerated decline of performance with increasing memory demand, in accord with previous data from the memoryupdating task (Oberauer \& Kliegl, 2001). Contrary to the previous results, the decline was not steeper for old than for young adults.

The main analysis focused on the latencies of individual updating operations (i.e., times between two hits on the space bar), because only these could reflect differences between switch and nonswitch operations. Data were only included from trials on which all final values were recalled correctly, which implies that every individual operation was performed correctly. To control for outliers, we computed individual means and standard deviations within the design cells formed by crossing memory demand and object switch; all latencies exceeding an individual's cell mean by more than three standard deviations were removed (1.5\% of all observations). The resulting mean latencies are summarized in Table 2.

To test the effect of task complexity (i.e., memory demand) independently of object switching, we submitted the nonswitch latencies to an ANOVA, with memory demand (1 to 4 ) and age as factors. There was a main effect of memory demand $\left[F(1.2,55.2)=88.78, M S_{\mathrm{e}}=0.65\right]$ (noninteger $d f$ s are due to Greenhouse-Geisser correction). The main effect of age was not significant $(F=$ $1.90)$. The critical interaction of age and memory demand just failed the significance level $[F(1.2,55.2)=2.64, p=$ .10]. This was due to one young participant who had exceptionally large mean latencies overall $(5.4 \mathrm{sec}$, which is 3.7 SDs above the mean of the young group and 3.4 SDs above the mean of the old group). When the analysis was repeated with this participant excluded, ${ }^{1}$ the main effect of age was significant $\left[F(1,45)=4.94, M S_{\mathrm{e}}=1.66\right]$, as was the interaction $\left[F(1.3,59.2)=7.11, M S_{\mathrm{e}}=0.39\right]$.

We repeated the same ANOVA with log-transformed latencies. This is the more critical test of the hypothesis that old adults have a specific impairment in tasks with coordinative complexity, one that goes beyond general slowing. An interaction of age with memory demand on untransformed latencies would be expected from proportional slowing alone. The log transformation translates proportional slowing effects into mere additive effects of experimental factors with age. Interactions on log-transformed data, therefore, are clear evidence that an experimental factor such as memory demand is associated with a specific age deficit over and above general slowing (see Cerella, 1990; Kliegl et al., 1994). With log-transformed

Table 2

Mean Latencies (RT, in Milliseconds) for Young and Old Participants, With Standard Deviations

\begin{tabular}{|c|c|c|c|c|c|c|c|c|}
\hline \multirow{3}{*}{$\begin{array}{l}\text { Memory } \\
\text { Demand }\end{array}$} & \multicolumn{4}{|c|}{ Young } & \multicolumn{4}{|c|}{ Old } \\
\hline & \multicolumn{2}{|c|}{ Nonswitch } & \multicolumn{2}{|c|}{ Switch } & \multicolumn{2}{|c|}{ Nonswitch } & \multicolumn{2}{|c|}{ Switch } \\
\hline & RT & $S D$ & RT & $S D$ & RT & $S D$ & RT & $S D$ \\
\hline 1 & 1,351 & 303 & & & 1,347 & 334 & & \\
\hline 2 & 1,643 & 516 & 1,961 & 597 & 1,867 & 670 & 2,093 & 681 \\
\hline 3 & 1,992 & 869 & 2,440 & 1,009 & 2,472 & 989 & 2,869 & 952 \\
\hline 4 & 2,711 & 1,350 & 3,491 & 1,545 & 3,212 & 1,277 & 3,841 & 1,337 \\
\hline
\end{tabular}


latencies, the interaction of age and memory demand was again significant (even without excluding the outlier) $\left[F(1.6,77.3)=6.88, M S_{\mathrm{e}}=0.03\right]$.

Figure 3 and Table 2 show that young and old adults had equivalent latencies in the sequentially complex condition; the age difference for memory demand 1 did not approach significance even after removal of the slow young outlier $(t=.17)$. In contrast, old adults were slower than young in the conditions with coordinative complexity (i.e., memory demands $>1$ ). This pattern replicates the disordinal interaction of age and type of task complexity observed by Verhaeghen et al. (1997).

Next, we investigated age effects on access to working memory in an ANOVA with memory demand (2 to 4), age, and object switching (switch vs. no switch) as factors. The main effect of memory demand was again significant $\left[F(1.1,50.6)=166.1, M S_{\mathrm{e}}=0.87\right]$, but it did not interact with age $(F=1.2)$. More important, there was a reliable main effect of object switching $[F(1,46)=166.1$, $\left.M S_{\mathrm{e}}=0.09\right]$. The object switch effect increased with larger memory demands, as was indicated by a reliable two-way interaction $\left[F(1.3,58.2)=43.47, M S_{\mathrm{e}}=0.04\right]$. The interaction of object switching with age was not significant $(F=1.8)$; the mean switch costs of the old group were numerically smaller. The object-switching costs, computed by subtracting nonswitch from switch latencies in each memory demand condition, are shown as columns in Figure 3.

Unsurprisingly, the time taken to initially encode the digits increased with increases in memory demand, with a slope close to one $\left[F(3,138)=87.6, M S_{\mathrm{e}}=2.5\right]$. Young adults took a mean of $1.05 \mathrm{sec}$ per digit, whereas old adults took $1.74 \mathrm{sec}$, a significant difference $[F(1,46)=$ $\left.13.4, M S_{\mathrm{e}}=1.7\right]$. Could old adults have gained an advantage from the longer encoding times for the subsequent speed of executing arithmetic operations? This is unlikely, given that the initial digits were quickly replaced by new ones. If anything, such an advantage would soon have worn out over the sequence of 13 operations, leading to an increase in latencies within a trial. We correlated latencies of switch and nonswitch operations with their serial position in the operation sequence (1-13). Old adults became slightly faster over the course of a trial in the nonswitch operations $(r=-.06$, slope $=-18 \mathrm{msec})$, and young adults slowed down on switch operations $(r=.03$, slope $=$ $16 \mathrm{msec})$. The other two correlations were $<.01$. Therefore, there is little evidence that the quality of initial encoding affected processing latencies.

We used a final analysis to investigate the problem-size effect for updating operations as a function of age and memory demand. We computed an ANOVA with age (2), memory demand (4), type of arithmetic operation (addition vs. subtraction), and absolute value of the operand (7) as factors and operation latencies as the dependent variable. The linear contrast of the absolute value of the operand was significant $\left[F(1,44)=47.9, M S_{\mathrm{e}}=0.35\right]$, reflecting an increase of reaction times over problem size with a slope of $41 \mathrm{msec}$. The linear trend of operand value interacted with the linear trend of memory demand $\left[F(1,44)=54.4, M S_{\mathrm{e}}=0.21\right]$, indicating a monotonic increase of the problem-size effect with increasing memory demand (means of participants' slopes were $-5,33,34$, and $84 \mathrm{msec}$ for memory demands 1, 2, 3, and 4, respectively). The problem-size contrast, however, did not interact with age $(F<1)$.

\section{DISCUSSION}

The results of our study confirm the dissociation of two classes of tasks, those with purely sequential complexity and those with coordinative complexity, for the domain of mental arithmetic (Verhaeghen et al., 1997). In tasks with

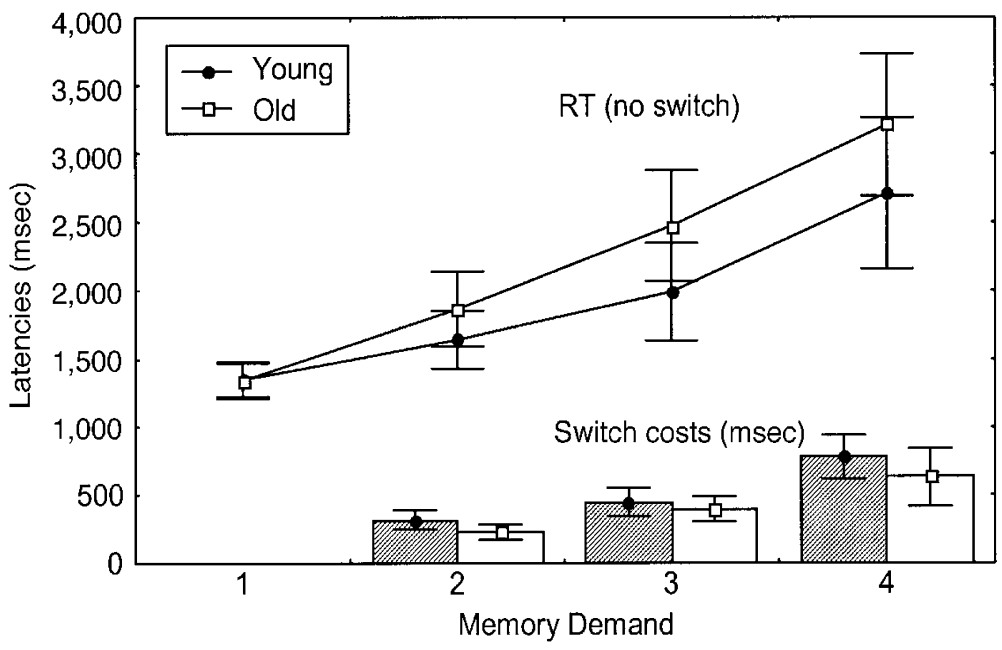

Figure 3. Mean latencies for young and old adults on nonswitch trials (dots connected by lines) and switch costs (hatched columns = young adults; white columns = old adults). Error bars represent two standard errors. Mean latencies in switch trials can be obtained by adding switch costs to the nonswitch means. 
sequential complexity, old and young adults performed equivalently with respect to both mean latencies and accuracy. In contrast, age differences in latencies emerged for tasks with coordinative complexity. Such a disordinal interaction is difficult to explain for any theory that proposes that age effects can be reduced to a single factor. Age effects in the coordinative tasks can hardly be explained by age differences in basic processes that are somehow magnified in the coordinative conditions (Salthouse, 1996; Salthouse \& Coon, 1994), because age differences in basic processes should have led to measurable, if only small, differences in the sequential condition as well. The old participants took more time for encoding of the initial items, but any advantage they might have gained from this would soon be lost because of the replacement of these items through the updating operations. Still, old adults performed simple arithmetic operations as fast as young adults.

What is the critical difference between sequential and coordinative complexity? In this study, we contrasted two hypotheses: (1) Old adults have specific problems with holding more than one element at a time available in working memory, and (2) old adults have specific difficulties with selective access to an element in working memory.

The results regarding these hypotheses were clear: Age differences in latencies for arithmetic operations increased with increases in memory demand, even when no new element had to be accessed to perform an operation, thus supporting the first hypothesis. Contrary to the second hypothesis, the costs for accessing a new object in working memory were not enlarged in older age. If anything, the old adults had smaller object switch costs than did the young adults. This is in good agreement with the conclusions of Oberauer et al. (2001), who found that old adults had no particular problems with accessing information in working memory. It is also consistent with a study from Belleville, Rouleau, and Caza (1998), who found that after equating young and old adults in their performance on a serial recall task, adding a processing demand that required selective access to elements of the memory set (reordering the letters in alphabetical order) did not impair the old participants more than the young. Remarkably, the object switch costs of the old adults were even less than would be expected from proportional slowing. This could indicate that access to working memory is a process unaffected by aging at all, thereby constituting another example of age invariance in a specific cognitive process.

It is noticeable that age differences in the present study, even in the coordinative conditions, were only moderate, in comparison with what was found for tasks with coordinative complexity in previous studies (Kliegl et al., 1994; Mayr \& Kliegl, 1993; Mayr et al., 1996). Old-young ratios for mean latencies were $1.14,1.24$, and 1.18 for memory demands 2,3 , and 4 , respectively. This might be due in part to the specific samples in the present study; in very similar arithmetic tasks used by Oberauer et al. (2001) and Oberauer and Kliegl (2001), the age differences were considerably larger (but see Kliegl et al., 2000, for another example of nearly equivalent performance).
The small slowing ratios, however, are in line with accumulating evidence that age effects are minimal in tasks based mainly on retrieval of lexical knowledge from longterm memory (Jenkins, Myerson, Joerding, \& Hale, 2000; Lima, Hale, \& Myerson, 1991; Mayr \& Kliegl, 2000; Verhaeghen et al., 1997). In addition, old adults' strategy mix has been found to include a smaller proportion of slow algorithmic responses and a larger proportion of direct retrievals (Geary \& Wiley, 1991). Since direct retrieval is usually faster, this would also contribute to shorter mean latencies than expected from the speed of basic processes. An age difference in strategy mix, however, is unlikely to contribute much to the present results, because a relatively high proportion of nonretrieval strategies was observed in young adults only for equations with results larger than 10 , which were excluded in the present study. For equations with results $<10$, even young adults reported use of retrieval for $90 \%$ of their responses (LeFevre, Sadesky, \& Bisanz, 1996). Age invariance in arithmetic fact retrieval is also supported by the lack of an interaction of age with the problem-size effect. This has been found consistently now in a number of studies (Allen et al., 1992; Allen et al., 1997; Geary, Frensch, \& Wiley, 1993; Geary \& Lin, 1998; Geary \& Wiley, 1991; Sliwinski et al., 1994).

Problem size did interact, however, with memory demand, and this seems to contrast with previous reports that arithmetic fact retrieval is unimpaired by a load on working memory (Ashcraft, Donley, Halas, \& Vakali, 1992; Hecht, 2002). In a dual-task study with young adults, Hecht found that two secondary tasks - articulatory suppression and random letter generation - increased the problem-size effect only for trials on which participants reported counting, not retrieval, as their strategy. The secondary tasks also did not affect the strategy mix. One potentially important difference between the present study and Hecht's is that we manipulated working memory load by increasing the number of elements kept available as potential inputs to the arithmetic operations. Oberauer et al. (2001) showed that the speed of executing self-paced arithmetic operations is affected by an additional working memory load only if the material held in working memory has to be accessed as input for the operations. One potential explanation for this is that an increase in the number of elements held in working memory degrades the representation of each element, thereby reducing the quality of the input to cognitive operations performed on them. This would slow down only the concurrent processes that require these representations as input.

As a by-product, the overall pattern of results obtained in this experiment questions the equivalence of aging and putting a higher load on working memory. A common assumption in the literature on cognitive capacity is that placing a higher load on working memory reduces the resources available for additional storage or processing (e.g., Just \& Carpenter, 1992). Likewise, getting older is assumed to reduce cognitive resources (e.g., Salthouse, 1988). In a previous paper (Oberauer \& Kliegl, 2001), we assumed for simplicity that aging and increasing working memory load affect the same parameter in a family of 
mathematical models of working memory capacity. The experiment reported here showed that increasing the load on working memory strongly affected object switch costs, whereas aging had no such effect at all. Likewise, memory load affected the problem size effect, whereas age did not. Accommodating these findings will require a more complex model than we envisaged before.

To summarize, our study provides a clear dissociation of age differences and age invariances. Basic mental arithmetic processes were hardly affected by aging. This adds to a growing body of research showing preserved numerical abilities in old adults, calling into question the hypothesis of age-related general slowing across all domains. Increasing the load on working memory resulted in a small, but reliable age-related decline in performance, corroborating the distinction between sequential and coordinative complexity (Mayr \& Kliegl, 1993). On the other hand, no age deficits were observed in the speed of accessing an element in working memory. This allows us to characterize age-related deficits in working memory more precisely than before: The source of the deficit is most likely the ability to keep elements accessible in working memory, not to access them efficiently.

\section{REFERENCES}

Allen, P. A., Ashcraft, M. H., \& Weber, T. A. (1992). On multiplication and age. Psychology \& Aging, 7, 536-545.

Allen, P. A., Smith, A. F., Jerge, K. A., \& Vires-Collins, H. (1997). Age differences in mental multiplication: Evidence for peripheral but not central decrements. Journals of Gerontology, 52B, P81-P90.

Ashcraft, M. H., Donley, R. D., Halas, M. A., \& VaKali, M. (1992). Working memory, automaticity, and problem difficulty. In J. I. D. Campbell (Ed.), The nature and origins of mathematical skill (pp. 301-329). Amsterdam: Elsevier.

BAdDeley, A. D. (1986). Working memory. Oxford: Oxford University Press.

BADDELEY, A. D. (1996). Exploring the central executive. Quarterly Journal of Experimental Psychology, 49A, 5-28.

Belleville, S., Rouleau, N., \& Caza, N. (1998). Effect of normal aging on the manipulation of information in working memory. Memory \& Cognition, 26, 572-583.

Cerella, J. (1990). Aging and information processing rate. In J. E. Birren \& K. W. Schaie (Eds.), Handbook of the psychology of aging (3rd ed., pp. 201-221). San Diego: Academic Press.

CowAN, N. (1999). An embedded process model of working memory. In A. Miyake \& P. Shah (Eds.), Models of working memory (pp. 62-101). Cambridge: Cambridge University Press.

Craik, F. I. M., Anderson, N. D., Kerr, S. A., \& Li, K. Z. H. (1995). Memory changes in normal ageing. In A. D. Baddeley, B. A. Wilson, \& F. N. Watts (Eds.), Handbook of memory disorders (pp. 211-241). Chichester, U.K.: Wiley.

Engle, R. W., Tuholski, S. W., Laughlin, J. E., \& Conway, A. R. A. (1999). Working memory, short-term memory and general fluid intelligence: A latent-variable approach. Journal of Experimental Psychology: General, 128, 309-331.

FisK, J. E., \& WARR, P. (1996). Age and working memory: The role of perceptual speed, the central executive, and the phonological loop. Psychology \& Aging, 11, 316-323.

GaRAVAN, H. (1998). Serial attention within working memory. Memory \& Cognition, 26, 263-276.

Geary,D. C., Frensch, P. A., \& WiLEy, J. G. (1993). Simple and complex mental subtraction: Strategy choice and speed-of-processing differences in younger and older adults. Psychology \& Aging, 8, 242-256.

GEARY, D. C., \& LiN, J. (1998). Numerical cognition: Age-related differences in the speed of executing biologically primary and biologically secondary processes. Experimental Aging Research, 24, 101-137.
Geary, D. C., \& Wiley, J. G. (1991). Cognitive addition: Strategy choice and speed-of-processing differences in young and elderly adults. Psychology \& Aging, 6, 474-483.

HECHT, S. A. (2002). Counting on working memory in simple arithmetic when counting is used for problem solving. Memory \& Cognition, $\mathbf{3 0}$, 447-455.

Jenkins, L., Myerson, J., Joerding, J. A., \& Hale, S. (2000). Converging evidence that visuospatial cognition is more age-sensitive than verbal cognition. Psychology \& Aging, 15, 157-175.

Just, M. A., \& CARPENTER, P. A. (1992). A capacity theory of comprehension: Individual differences in working memory. Psychological Review, 99, 122-149.

Kliegl, R., Mayr, U., \& Krampe, R.T. (1994). Time-accuracy functions for determining process and person differences: An application to cognitive aging. Cognitive Psychology, 26, 134-164.

Kliegl, R. Mayr, U., \& Oberauer, K. (2000). Resource limitations and process dissociations in individualdifferences research. In U. von Hecker, S. Dutke, \& G. Sedek (Eds.), Generative mental processes and cognitive resources: Integrative research on adaptation and control (pp. 337-366). Dordrecht: Kluwer.

LeFevre, J.-A., SADESKy, G. S., \& BisAnz, J. (1996). Selection of procedures in mental addition: Reassessing the problem size effect in adults. Journal of Experimental Psychology: Learning, Memory, \& Cognition, 22, 216-230.

Lima, S. D., Hale, S., \& Myerson, J. (1991). How general is general slowing? Evidence from the lexical domain. Psychology \& Aging, 6, 416-425.

MAYR, U., \& KLIEgL, R. (1993). Sequential and coordinative complexity: Age-based processing limitations in figural transformations. Journal of Experimental Psychology: Learning, Memory, \& Cognition, 19, 1297-1320.

MaYr, U., \& KLIEgl, R. (2000). Complex semantic processing in old age: Does it stay or does it go? Psychology \& Aging, 15, 29-43.

MaYr, U., KLIEgL, R., \& Krampe, R. T. (1996). Sequential and coordinative processing dynamics in figural transformation across the life span. Cognition, 59, 61-90.

OBERAUER, K. (2002). Access to information in working memory: Exploring the focus of attention. Journal of Experimental Psychology: Learning, Memory, \& Cognition, 28, 411-421.

Oberauer, K., Demmrich, A., Mayr, U., \& Kliegl, R. (2001). Dissociating retention and access in working memory: An age-comparative study of mental arithmetic. Memory \& Cognition, 29, 18-33.

Oberauer, K., \& Kliegl, R. (2001). Beyond resources: Formal models of complexity effects and age differences in working memory. European Journal of Cognitive Psychology, 13, 187-215.

Salthouse, T. A. (1988). Resource-reduction interpretations of cognitive aging. Developmental Review, 8, 238-272.

Salthouse, T. A. (1994). The aging of working memory. Neuropsychology, 8, 535-543.

SAlthouse, T. A. (1996). The processing speed theory of adult age differences in cognition. Psychological Review, 103, 403-428.

Salthouse, T. A., \& Coon, V. E. (1994). Interpretation of differential deficits: The case of aging and mental arithmetic. Journal of Experimental Psychology: Learning, Memory, \& Cognition, 20, 1172-1182.

Sliwinski, M., BuschKe, H., Kuslansky, G., Senior, G., \& ScarisBRICK, D. (1994). Proportional slowing and addition speed in old and young adults. Psychology \& Aging, 9, 72-80.

Verhaeghen, P., Kliegl, R, \& Mayr, U. (1997). Sequential and coordinative complexity in time-accuracy functions for mental arithmetic. Psychology \& Aging, 12, 555-564.

\section{NOTE}

1. All other analyses were also computed with the outlier removed. This didn't change any of the conclusions, so we report only the results from the full sample.

(Manuscript received October 4, 2002; revision accepted for publication February 5, 2003.) 